\title{
THE DISCRETE FRACTIONAL FOURIER TRANSFORM AND HARPER'S EQUATION
}

\author{
LAURENCE BARKER
}

Abstract. It is shown that the discrete fractional Fourier transform recovers the continuum fractional Fourier transform via a limiting process whereby inner products are preserved.

\$1. Discussion. A generalization of the Fourier Transform (FT), the fractional FT of Namias [13], is widely used in signal-processing, optics, and quantum mechanics; see Özaktaş-Mendlovic-Kutay-Zalevsky [15] for discussions of applications and history. The discrete FT, already a valuable tool in classical number theory during the 1930's, acquired, with the advent of computers, a tremendous range of applications throughout science and engineering; any calculation executed by a digital machine is, by nature, discrete. Pei-Yeh [16] introduced the discrete fractional FT. Candan [8] proved that the discrete fractional FT is uniquely determined by its defining properties; see CandanKutay-Özaktaş [9]. The eigenvectors of the discrete fractional FT, which we call the Harper functions, "converge" to the eigenvectors of the fractional FT, which are the Hermite Gaussian functions. As operators, the discrete fractional FT "converges" to the fractional FT. Numerical evidence for these "convergences" was given in [16], and a heuristic argument was given in [8] and [9].

We have been unable to consolidate Candan's heuristic argument. Our proof of the above "convergence" property is along different lines. The crux of the matter is the formulation of a suitable notion of "convergence". Actually, we feel that "convergence" is an inappropriate name, because that which is in question is an infinite sequence whose terms and whose "limit" all belong to different spaces. Now, an important feature of both the discrete fractional FT and the continuum fractional FT is that they are unitary operators. This being so, we must recognize that the spaces upon which they act are inner product spaces. The notion of induction, introduced below, is intended to describe a discrete to continuum "limiting" process that preserves inner products. A more general and systematic examination of induction, together with further examples, may be found in [6]. Some applications of the induction property of the fractional FT are described in [7]. In this paper-in Theorems 2.5 and 2.8, respectively-we show that the Harper functions induce the Hermite-Gaussians, and that the discrete fractional FT induces the fractional FT.

Athanasiu-Floratos [1], Athanasiu-Floratos-Nicolis [2], Balian-Itzykson [4] and Hakioğlu [10] have studied discrete quantum systems that admit a symmetry under the action of a suitable one-parameter group of linear canonical transforms. Independently, in the context of discrete time-frequency analysis, Richman-Parks-Shenoy [19] pursued similar ideas, and in further detail. 
They showed that, just as in the continuum scenario, the actions of the linear canonical transforms on signal space and phase space are covariant with the Weyl-Wigner correspondence. Some extensions of their results appear in [5]. At the time of writing, it remains unclear as to whether or not there is any general correspondence between some appropriate group of discrete linear canonical transforms and the group of continuum linear canonical transforms (a correspondence whereby "taking the limit as $n \rightarrow \infty$ " does not just mean taking the analogy with $n$ replaced by $\infty$.) It is reassuring that such a correspondence does exist for the discrete and continuum fractional FT's (the continuum FT's comprise a one-parameter subgroup of the group of continuum linear canonical transforms).

To make our results (if not all their proofs) accessible to a broad range of scientists, we have occasionally leaned towards pedagogy. On the other hand, no knowledge of science is needed to understand the results and their proofs. Yet part of the fascination of the discrete fractional FT comes from a dialogue it stimulates between mathematics and physics. In these introductory comments on motivation, it would be philistine not to indicate the ways in which Harper's Equation provides a rapport between the material in this paper and two separate topics in quantum mechanics. Consider an integer $n \geqslant 1$, let $\psi$ be a function $\mathbb{Z} \rightarrow \mathbb{C}$ with period dividing $n$, and let $E \in \mathbb{R}$. Harper's Equation is

$$
\psi(x-1)+2 \cos (2 \pi x / n) \psi(x)+\psi(x+1)=(4-2 \pi E / n) \psi(x),
$$

where $x \in \mathbb{Z}$. Assume now that $\psi$ and $E$ are solutions to Harper's Equation. Then $\psi$ is a Hamiltonian eigenstate for a discrete analogue of a quantum harmonic oscillator. To see this in a quick informal way, take $n$ to be large, write $\xi=x \sqrt{2 \pi / n}$, and suppose that $\xi \ll \sqrt{n}$. Put

$$
\varphi(\xi)=(n / 2 \pi)^{1 / 4} \psi(x) .
$$

(The factor $(n / 2 \pi)^{1 / 4}$ is just for normalization, and can be ignored for the purpose of the present discussion.) Then Harper's Equation reduces to

$$
n / 2 \pi(-\varphi(\xi-\sqrt{2 \pi / n})+2 \varphi(\xi)-\varphi(\xi+\sqrt{2 \pi / n}))+\xi^{2} \varphi(\xi)=E \varphi(\xi)+O\left((\xi / n)^{2}\right) .
$$

Thus we have obtained an approximation to a difference analogue of the Schrödinger equation

$$
\left(-\frac{d^{2}}{d r^{2}}+r^{2}-E\right) \theta(r)=0
$$

of a harmonic oscillator. The Hamiltonian eigenstates of this harmonic oscillator are the solutions $\theta=h_{j}$, where $h_{j}$ is the $j$-th Hermite Gaussian function $\mathbb{R} \rightarrow \mathbb{C}$ (but with real values) given by

$$
h_{j}(r)=\pi^{-1 / 4} 2^{-j / 2}(j !)^{-1 / 2} e^{-r^{2}} H_{j}(r),
$$

and $H_{j}$ is the Hermite polynomial of degree $j$ given by

$$
H_{j}(r)=(-1)^{j} e^{r^{2}} \frac{d^{j}}{d r^{j}} e^{-r^{2}}
$$


Here, the index $j$ runs over the set $\mathbb{N}=\{0,1,2, \ldots\}$ of natural numbers together with 0 . The energy eigenvalue corresponding to the eigenstate $h_{j}$ is $2 j+1$. In other words,

$$
\left(-\frac{d^{2}}{d r^{2}}+r^{2}\right) h_{j}(r)=(2 j+1) h_{j}(r) .
$$

In anticipation of a comment below, we recall that the above Schrödinger equation also has a family of "physically uninteresting" solutions which behave as $e^{r^{2} / 2}$. We also mention that the time-evolution operator of the above harmonic oscillator is a (time-dependent) scalar multiple of the fractional FT.

In Atakishiev-Suslov [3], two other discrete analogues of a harmonic oscillator are examined in connection with two other discrete analogues of the Hermite-Gaussians, namely, the Kravchuk polynomials and the Hermite $q$ polynomials. It is an open question as to what relationship, if any, exists between the Harper functions and either the Kravchuk polynomials or the Hermite q-polynomials.

For a Bloch electron confined to the lattice $\mathbb{Z} \times \mathbb{Z}=\{(a, b): a, b \in \mathbb{Z}\}$ and subject to a uniform transverse time-invariant magnetic field, let us impose the Landau gauge. Then the Hamiltonian $\hat{h}$ acts on a wave function $\theta$ such that

$$
(\hat{h} \theta)(a, b)=\theta(a-1, b)+\theta(a+1, b)+e^{-2 \pi i \omega \alpha} \theta(a, b-1)+e^{2 \pi i \omega \alpha} \theta(a, b+1),
$$

where $\omega$ is a real constant proportional to the magnetic flux. To obtain periodic solutions, let us assume that $\omega$ is rational, and write $\omega=m / n$ where $m$ and $n$ are coprime integers, and $n \geqslant 1$. For the Schrödinger equation $\hat{h} \theta=H \theta$, the $n^{2}$ solutions with period dividing $n$ coincide with the solutions to

$$
\theta(a, b)=e^{2 \pi i k b / n} \varphi(a)
$$

where $k$ is an integer, and

$$
\varphi(a-1)+\varphi(a+1)+2 \cos (2 \pi(m a+k) / n) \varphi(a)=H \varphi(a) .
$$

This form of Harper's Equation is essentially no more general than before. Indeed, since $m$ is coprime to $n$, the change of variables $a \rightarrow m a+k$ is invertible up to congruence modulo $n$, and we can put $\psi(m a+k)=\varphi(a)$ and $E=$ $n(4-H) / 2 \pi$. For a study of Bloch electrons subjected to uniform magnetic fields see, for instance, Rammal-Bellissard [18].

Returning to purely mathematical considerations, Harper's Equation (as an abstract system of linear equations) is the starting point for the Pei-Yeh definition of the discrete fractional FT. The Harper functions are defined to be precisely those solutions to Harper's Equation which commute with the discrete FT; see Section 2. Theorem 2.5, below, may be interpreted as saying that, for fixed $F \in \mathbb{R}$, and as $n$ increases, the Harper functions with eigenvalues at least $4-2 \pi F / n$ induce the Hermite-Gaussians. This conclusion was perhaps to be expected in view of the physics above, and we have already indicated the numerical and heuristic evidence. Without Theorem 2.5, how could we be sure that no Harper functions with high eigenvalues induce the "physically uninteresting" solutions to the Schrödinger equation of the above harmonical oscillator? Admittedly, this particular query can easily be resolved using 
Lemma 3.4. But how could we be sure that, for large $n$, there are no Harper functions which have high eigenvalues, yet have nothing to do with the Hermite-Gaussians?

For an integer $n \geqslant 1$, let $L(\mathbb{Z}(n))$ denote the space of functions $\psi: \mathbb{Z} \rightarrow \mathbb{C}$ such that $\psi(x+n)=\psi(x)$ for all $x \in \mathbb{Z}$. Let $\mathbb{Z}(n)$ denote the set consisting of the integers $x$ such that $-n / 2<x \leqslant n / 2$. We define addition on $\mathbb{Z}(n)$ so as to respect the usual addition in $\mathbb{Z}$ up to congruence modulo $n$. Thus, given $x, y, z \in \mathbb{Z}(n)$, then $x+y=z$ provided $n$ divides $x+y-z$. (For some of our purposes, it would be more elegant to replace $\mathbb{Z}(n)$ with the ring of modulo $n$ residue classes. However, for technical reasons, it will be more convenient to work with the fixed set $\mathbb{Z}(n)$ of representatives of the residue classes.) We define an inner product on $L(\mathbb{Z}(n))$ by

$$
\langle\varphi \mid \psi\rangle:=\sum_{x \in \mathbb{Z}(n)} \varphi(x)^{*} \psi(x) .
$$

We shall establish some connections between a continuum scenario based on the complex Hilbert space $L^{2}(\mathbb{R})$ and discrete scenarios based on the finitedimensional spaces $L(\mathbb{Z}(n))$ in the limit as $n \rightarrow \infty$. Some scaling factors will be involved, and it is convenient to define

$$
v(n):=(n / 2 \pi)^{1 / 4} \text {. }
$$

The period $n$ will not be allowed to range over all of $\mathbb{N}$. We shall confine $n$ to an infinite subset $\ldots \subset \mathbb{N}$ satisfying a suitable hypothesis. Vectors in $L^{2}(\mathbb{R})$ are to be related to some sequences of the form $\left(\psi_{n}: n \in 1\right)$, where each $\psi_{n} \in$ $L(\mathbb{Z}(n))$. Operators on $L^{2}(\mathbb{R})$ are to be related to some sequences of the form $\left(\hat{a}_{n}: n \in A\right)$, where each $a_{n}$ is an operator on $L(\mathbb{Z}(n))$. Some technical care will be needed, and in order that the wood not be lost for the trees, let us indicate, in a casual way, the rough idea. Consider a vector $\psi \in L^{2}(\mathbb{R})$, and for simplicity of discussion, let us assume that $\psi$ is rapidly decreasing. Loosely speaking, a sequence $\left(\psi_{n}: n \in \mathcal{A}\right)$ with $\psi_{n} \in L(\mathbb{Z}(n))$ will be deemed to be related to the vector $\psi$ provided that we have an approximate equality

$$
\psi\left(x v(n)^{-2}\right) \simeq v(n) \psi_{n}(x)
$$

for large $n$ and for most of the elements $x \in \mathbb{Z}(n)$. The analogous relationship between operators will be constructed in such a way as to respect the relationship between vectors. This idea, in some form or another, has always been used in realizations of the FT as a continuum limit of the discrete FT. In Section 2, we express this idea in an algebraic way that will suit our purposes here and in subsequent work. We also review the Pei-Yeh definition of the discrete fractional FT, and we state our main results, Theorems 2.5 and 2.8 . The four theorems stated in Section 2 are proved in Section 3.

§2. Results. Recall that a smooth function $\varphi: \mathbb{R} \rightarrow \mathbb{C}$ is said to be rapidly decreasing provided, for all $a, b \in \mathbb{N}$, the function $\mathbb{R} \rightarrow \mathbb{R}$ given by $r \mapsto\left|\varphi^{(a)}(r) r^{b}\right|$ has an upper bound. A dense subspace of $L^{2}(\mathbb{R})$, the $S$ chwartz space $y(\mathbb{R})$ is defined to be the space of rapidly-decreasing functions. It is well-known that 
the set $\left\{h_{j}: j \in \mathbb{N}\right\}$ of Hermite-Gaussians is a complete set in $(\mathbb{R})$, and is an orthonormal basis for the Hilbert space $L^{2}(\mathbb{R})$. Following Namias [13], we define, for each $t \in \mathbb{R}$, an operator $\hat{f}^{[t]}$ on $L^{2}(\mathbb{R})$ given by

$$
\hat{f}^{[t]} h_{j}=e^{2 \pi i j t} h_{j} .
$$

Namias showed that, for $0<t<1 / 2$ and $\varphi \in,(\mathbb{R})$, we have

$$
\left(\hat{f}^{|t|} \varphi\right)(r)=(2 \pi \sin 2 \pi t)^{-1 / 2} \int_{-\infty}^{\infty} K_{t}(r, q) \varphi(q) d q,
$$

where the kernel is

$$
K_{t}(r, q)=\exp \left(i \pi(t-1 / 4)-i(\cot 2 \pi t)\left(r^{2}+q^{2}\right) / 2+i r / \sin 2 \pi t\right) .
$$

As observed in McBride-Kerr [11], the action of $\hat{f}^{[t]}$ on,$(\mathbb{R})$ for arbitrary $t \in$ $\mathbb{R}$ is determined by the explicit integral formula together with the condition that $\hat{f}^{\left[t+t^{\prime}\right]}=\hat{f}^{[t]} \hat{f}^{\left[i^{\prime}\right]}$ for all $t, t^{\prime} \in \mathbb{R}$. The Fourier Transform $\hat{f}$ on $L^{2}(\mathbb{R})$ is the special case

$$
\hat{f}=\hat{f}^{[1 / 4]},
$$

so it makes sense to call $\hat{f}^{[t]}$ the fractional Fourier Transform.

The operator on $1 /(\mathbb{R})$ given by $\varphi(r) \mapsto\left(-d^{2} / d r^{2}+r^{2}\right) \varphi(r)$ extends to an operator $\hat{h}$ on $L^{2}(\mathbb{R})$, given by

$$
\hat{h} h_{j}=(2 j+1) h_{j}
$$

for $j \in \mathbb{N}$. Evidently, $\hat{f}^{[t]}=\exp (i \pi t(\hat{h}-1))$.

Consider an integer $n \geqslant 1$. For the moment, let us take $n$ to be fixed. Recall that the discrete Fourier Transform $\hat{f}_{n}$ is defined to be the operator on $L(\mathbb{Z}(n))$ such that

$$
\left(\hat{f}_{n} \psi\right)(x)=\frac{1}{\sqrt{n}} \sum_{y \in \mathbb{Z}(n)} e^{2 \pi i x y / n} \psi(y),
$$

where $\psi \in L(\mathbb{Z}(n))$ and $x \in \mathbb{Z}$. For each integer $x$, we write $x^{*}$ to denote the function $\mathbb{Z} \rightarrow \mathbb{C}$ such that $x^{*}(y)=1$ when $y \equiv x \bmod n$, and $x^{*}(y)=0$ otherwise. The set $\left\{x^{*}: x \in \mathbb{Z}(n)\right\}$ is an orthonormal basis for $L(\mathbb{Z}(n))$. It is easily shown that

$$
\hat{f}_{n} x^{*}=\frac{1}{\sqrt{n}} \sum_{y \in \mathbb{Z}(n)} e^{2 \pi i x y / n} y^{*} .
$$

Another easy calculation yields $\hat{f}_{n}^{2} x^{*}=(-x)^{*}$, whence $\hat{f}_{n}^{4}=1$.

Let $\hat{u}_{n}$ and $\hat{v}_{n}$ be the unitary operators on $L(\mathbb{Z}(n))$ such that $\hat{u}_{n} x^{*}=(x+1)^{*}$ and $\hat{v}_{n} x^{*}=e^{2 \pi i x / n} x^{*}$ for $x \in \mathbb{Z}$. The Hermitian conjugates satisfy $\hat{u}_{n}^{+} x^{*}=$ $(x-1)^{*}$ and $\hat{v}_{n}^{\dagger} x^{*}=e^{-2 \pi i x / n} x^{*}$. We define a Hermitian operator

$$
\hat{s}_{n}:=\hat{u}_{n}+\hat{u}_{n}^{\dagger}+\hat{v}_{n}+\hat{v}_{n}^{\dagger}
$$

on $L(\mathbb{Z}(n))$. Given an eigenvector $\sigma$ of $\hat{s}_{n}$ with corresponding eigenvalue $4-2 \pi E / n$, then $\sigma$ and $E$ satisfy Harper's Equation

$$
\sigma(x-1)+2 \cos (2 \pi x / n) \sigma(x)+\sigma(x+1)=(4-2 \pi E / n) \sigma(x) .
$$


REMARK 2.1. Let $\sigma$ be an eigenvector of $\hat{s}_{n}$. Then there does not exist an integer $x$ such that $\sigma(x)=0=\sigma(x+1)$.

Proof. This is immediate from the fact that $\sigma$ satisfies Harper's Equation.

THEOREM 2.2 (Candan [8]). Up to scalar multiplication, $L(\mathbb{Z}(n))$ has a unique basis consisting of common eigenvectors of $\hat{s}_{n}$ and $\hat{f}_{n}$.

Proof. We give a brief presentation of the argument in Candan-KutayOzaktas [9, Section III.B]. By direct calculation, $\hat{f}_{n} \hat{u}_{n} \hat{f}_{n}^{\dagger}=\hat{v}_{n}$ and $\hat{f}_{n} \hat{v}_{n} \hat{f}_{n}^{\dagger}=\hat{u}_{n}^{\dagger}$. Hence $\hat{f}_{n} \hat{s}_{n}=\hat{s}_{n} \hat{f}_{n}$. In particular, $L(\mathbb{Z}(n))$ has a basis consisting of common eigenvectors of $\hat{s}_{n}$ and $\hat{f}_{n}$. Let $L_{+}(\mathbb{Z}(n))$ and $L_{-}(\mathbb{Z}(n))$ be the eigenspaces of the involution $\hat{f}_{n}^{2}$ corresponding to the eigenvalues +1 and -1 , respectively. The action of $\hat{s}_{n}$ on $L(\mathbb{Z}(n))$ must respect the decomposition

$$
L(\mathbb{Z}(n))=L_{+}(\mathbb{Z}(n)) \oplus L_{-}(\mathbb{Z}(n)) .
$$

The sets $\left\{x^{*}+(-x)^{*}: x \in \mathbb{Z} \cap[0, n / 2]\right\}$ and $\left\{x^{*}-(-x)^{*}: x \in \mathbb{Z} \cap(0, n / 2)\right\}$ are bases for $L_{+}(\mathbb{Z}(n))$ and $L_{-}(\mathbb{Z}(n))$, respectively. With respect to these two bases, the actions of $\hat{s}_{n}$ on $L_{+}(\mathbb{Z}(n))$ and on $L_{-}(\mathbb{Z}(n))$ are both represented by tridiagonal matrices whose off-diagonal entries are all non-zero. By Wilkinson [20, Section 5.38], these two actions of $\hat{s}_{n}$ are both separable. Therefore, up to scalar multiplication, $L_{+}(\mathbb{Z}(n)) \cup L_{-}(\mathbb{Z}(n))$ contains a unique basis of eigenvectors of $\hat{s}_{n}$. The assertion follows because any eigenvector of $\hat{f}_{n}$ must belong either to $L_{+}(\mathbb{Z}(n))$ or else to $L_{-}(\mathbb{Z}(n))$.

By Remark 2.1, Theorem 2.2, and the fact that $\hat{s}_{n}$ is Hermitian, there exists a unique basis $S_{n}$ of $L(\mathbb{Z}(n))$ consisting of real unit eigenvectors $\sigma$ such that either $\sigma(0)>0$ or else $\sigma(0)=0<\sigma(1)$. Since the action of $\hat{s}_{n}$ on $L_{+}(\mathbb{Z}(n))$ is separable, we can enumerate the elements of $S_{n} \cap L_{+}(\mathbb{Z}(n))$ as $\sigma_{n, 0}$, $\sigma_{n, 2}, \sigma_{n, 4}, \ldots$, such that the corresponding eigenvalues are in strictly decreasing order. Similarly, we can enumerate the elements of $S_{n} \cap L(\mathbb{Z}(n))$ as $\sigma_{n, 1}, \sigma_{n, 3}, \sigma_{n, 5}, \ldots$, such that the corresponding eigenvalues are in strictly decreasing order. By considering the identity $n=|\mathbb{Z} \cap[0, n / 2]|+|\mathbb{Z} \cap(0, n / 2)|$, we see that $L_{+}(\mathbb{Z}(n)$ ) has dimension either $n / 2+1$ or $(n+1) / 2$ (whichever is an integer); likewise $L_{-}(\mathbb{Z}(n))$ has dimension either $n / 2-1$ or $(n-1) / 2$. Thus we have enumerated the elements $\sigma_{n, k}$ of $S_{n}$ such that, if $n$ is even, then the index $k$ is an integer satisfying $0 \leqslant k \leqslant n-2$ or $k=n$, while if $n$ is odd, then $k$ is an integer satisfying $0 \leqslant k \leqslant n-1$. After Candan [8], we define, for each $t \in \mathbb{R}$, the discrete fractional Fourier Transform $\hat{f}_{n}^{[t]}$ to be the operator on $L(\mathbb{Z}(n))$ such that

$$
\hat{f}_{n}^{[t]} \sigma_{n, k}:=e^{2 \pi i k t} \sigma_{n, k}
$$

for each index $k$ as above. Evidently, $\hat{f}_{n}^{\left[t+t^{\prime}\right]}=\hat{f}_{n}^{[t]} \hat{f}_{n}^{\left[t^{\prime}\right]}$ for all $t, t^{\prime} \in \mathbb{R}$. Note that $\hat{f}_{n}^{[1]}=1$. Furthermore, $\hat{f}_{n}^{[1 / 4]}$ has order 4 . Candan observed that, by McClellenParks [12], the multiplicities of $1, i,-1,-i$ as eigenvalues of $\hat{f}_{n}^{[1 / 4]}$ coincide with the multiplicities of $1, i,-1,-i$, respectively, as eigenvalues of $\hat{f}_{n}$. This, together with numerical evidence in Pei-Yeh [16] and Candan-KutayOzaktas [9] supports the following conjecture. 
CONJECTURE 2.A. Given an integer $n \geqslant 1$, then $\hat{f}_{n}^{[1 / 4]}=\hat{f}_{n}$.

Theorems 2.6 and 2.7, below, tell us that $\hat{f}_{n}^{[1 / 4]}$ and $\hat{f}_{n}$ both induce $\hat{f}$.

Consider a uniformly continuous vector $\varphi \in L^{2}(\mathbb{R})$. As in Section 1 , the unique function $\mathbb{R} \rightarrow \mathbb{C}$ representing $\varphi$ is also denoted by $\varphi$. Given a vector $\varphi_{n} \in L(\mathbb{Z}(n))$, we say that $\varphi$ restricts to $\varphi_{n}$, writing $\varphi_{n}=\operatorname{res}_{n}(\varphi)$, provided that, for all $x \in \mathbb{Z}(n)$, we have

$$
\varphi\left(x v(n)^{-2}\right)=v(n) \varphi_{n}(x) .
$$

Let, 1 be an infinite set of natural numbers such that, given $m, n \in f$ with $n \leqslant m$, then $n$ divides $m$, and the integer $m / n$ is a square. The set

$$
\mathbb{R}(1):=\left\{x v(n)^{-2}: n \in, ; x \in \mathbb{Z}(n)\right\}
$$

is dense in $\mathbb{R}$. Given an element $\xi \in \mathbb{R}(1)$, we define $\rho(\xi)$ to be the minimal element of 1 such that $\xi v(\rho(\xi))^{2} \in \mathbb{Z}(\rho(\xi))$. Thus, for each $n \in f$ with $n \geqslant \rho(\xi)$, we can write

$$
\xi=\xi(n) v(n)^{-2},
$$

where $\xi(n) \in \mathbb{Z}(n)$. Let $L(\mathbb{Z}(1))$ denote the space of sequences of the form $\left(\psi_{n}: n \in t^{\prime}\right)$ where each $\psi_{n} \in L(\mathbb{Z}(n))$. Since $\mathbb{R}(+)$ is dense in $\mathbb{R}$, each uniformly continuous vector $\psi \in L^{2}(\mathbb{R})$ is uniquely determined by the element $\left(\operatorname{res}_{n}(\psi): n \in 1\right)$ of $L(\mathbb{Z}(+))$. Note that any infinite subset $A^{\prime \prime}$ of $A$ satisfies the hypothesis we imposed upon $1^{\prime}$, and, furthermore, $\mathbb{R}\left(f^{\prime \prime}\right)=\mathbb{R}\left(A^{\prime}\right)$.

The condition on , that each integer $m / n$ be a square is not essential, and can be dispensed with, but at the price of introducing some irritating complications that would obscure our arguments. We do not know whether our results would still hold if. + were replaced with any infinite subset of $\mathbb{N}$. Anyway, the hypothesis we have imposed upon 1 covers all the applications we have in mind. As an example, we can take $A$ to consist of all the positive even powers of some given integer $d \geqslant 2$, or we can take to consist of all the positive odd powers of $d$. The case where $d$ is prime pertains to the references to discrete quantum mechanics cited in Section 1.

Consider a vector $\psi \in L^{2}(\mathbb{R})$, and a sequence $\left(\psi_{n}: n \in t\right) \in L(\mathbb{Z}(t))$. We say that $\left(\psi_{n}: n \in .1\right)$ induces $\psi$, writing $\psi=\operatorname{ind}_{n \in,}\left(\psi_{n}\right)$, provided that, for all uniformly continuous $\varphi \in L^{2}(\mathbb{R})$, we have

$$
\langle\varphi \mid \psi\rangle=\lim _{n \in .}\left\langle\operatorname{res}_{n}(\varphi) \mid \psi_{n}\right\rangle .
$$

By the Riesz Representation Theorem (for Hilbert spaces), each element of $L(\mathbb{Z}(+))$ induces at most one vector in $L^{2}(\mathbb{R})$.

LFMmA 2.3. Given uniformly continuous vectors $\varphi, \psi \in L^{2}(\mathbb{R})$, then

$$
\langle\varphi \mid \psi\rangle=\lim _{n \in,}\left\langle\operatorname{res}_{n}(\varphi) \mid \operatorname{res}_{n}(\psi)\right\rangle
$$


Proof. Let $\varphi_{n}:=\operatorname{res}_{n}(\varphi)$ and $\psi_{n}:=\operatorname{res}_{n}(\psi)$. Since $\varphi$ and $\psi$ are Riemannintegrable,

$$
\int_{-q}^{q} \varphi(r)^{*} \psi(r) d r=\lim _{n \in,} \sum_{x \in \mathbb{Z}(,):|x| \leqslant q v(n)^{2}} \varphi_{n}(x)^{*} \psi_{n}(x)
$$

for all real $q>0$. Via the Cauchy-Schwartz inequality, we easily obtain

$$
\left(\lim _{n \in,} \sum_{x \in \mathbb{Z}(n):|x|>q v(n)^{2}} \varphi_{n}(x)^{*} \psi_{n}(x)\right)^{2} \leqslant \int_{r:|r| \geqslant q}|\varphi(r)|^{2} d r \int_{r:|r| \geqslant q}|\psi(r)|^{2} d r .
$$

The right-hand expression tends to zero as $q$ attends to infinity.

LEMMA 2.4. Given a uniformly continuous vector $\psi \in L^{2}(\mathbb{R})$, then $\psi=$ ind $_{n \in,},\left(\operatorname{res}_{n}(\psi)\right)$.

Proof. This is immediate from Lemma 2.3.

The four theorems stated below will be proved in Section 3 .

TheOREM 2.5. For each $j \in \mathbb{N}, h_{j}=\operatorname{ind}_{n \in},\left(\sigma_{n, j}\right)$.

THEOREM 2.6. Given a vector $\psi \in L^{2}(\mathbb{R})$, then there exists an element $\left(\psi_{n}\right.$ : $n \in A) \in L(\mathbb{Z}(\mathcal{A}))$ such that $\psi=\operatorname{ind}_{n \in},(\psi(n))$. ring

For $n \in A$, let End $(L(\mathbb{Z}(n)))$ denote the ring of operators on $L(\mathbb{Z}(n))$. The

$$
\text { End }(L(\mathbb{Z}(+))):=X_{n \in,} \text { End }(L(\mathbb{Z}(n)))
$$

consists of the sequences $\left(\hat{a}_{n}: n \in \mathcal{A}\right)$ such that each $\hat{a}_{n} \in \operatorname{End}(L(\mathbb{Z}(n)))$. We say that $\left(\hat{a}_{n}: n \in, 1\right)$ induces $\hat{a}$, writing $\hat{a}=\operatorname{ind}_{n \in},\left(\hat{a}_{n}\right)$, provided that, given any element $\left(\psi_{n}: n \in \mathcal{A}\right) \in L(\mathbb{Z}(f))$ inducing a vector $\psi \in L^{2}(\mathbb{R})$, then $\left(\hat{a}_{n} \psi_{n}: n \in\right.$ . ) induces $\hat{a} \psi$. The Reisz Representation Theorem, together with Theorem 2.6, ensures that each element of End $(L(\mathbb{Z}(t)))$ induces at most one operator on $L^{2}(\mathbb{R})$.

The condition that $\left(\hat{a}_{n}: n \in t\right)$ induces $\hat{a}$ seems to be rather strong. Suppose, for instance, that each $a_{n}$ is given by

$$
\hat{a}_{n} x^{*}=v(n)^{2}\left((x-1)^{*}-2 x^{*}+(x+1)^{*}\right) .
$$

In numerical calculation, $\hat{a}_{n}$ is often used as a discrete approximation to the second differential operator. Of course, differential operators do not have domain $L^{2}(\mathbb{R})$, but that is not an essential problem, because we could easily extend the definition of induction so as to be applicable to operators on a domain dense in $L^{2}(\mathbb{R})$. Our point is that, putting $\psi_{n}(x)=(-1)^{x} n^{\gamma}$ with $\gamma \in \mathbb{R}$, then $\hat{a}_{n} \psi_{n}=-4 v(n)^{2} \psi_{n}$, and there clearly exist $\gamma$ for which $\left(\psi_{n}: n \in I\right)$ induces the zero-vector while $\left(\hat{a}_{n} \psi_{n}: n \in, 1\right)$ does not induce any vector. As another example, the operator $\hat{h}_{n}$ on $L(\mathbb{Z}(n))$ given by

$$
\hat{h}_{n} x^{*}=v(n)^{2}\left(-(x-1)^{*}+\left(4-2 \cos (2 \pi x / n) x^{*}-(x+1)^{*}\right)\right.
$$


may seem sensible as a discrete analogue of the operator $\hat{h}$ on $\gamma(\mathbb{R})$, but there does not exist a vector $\varphi \in L^{2}(\mathbb{R})$ such that $\left(h_{n} \varphi_{n}: n \in h^{\prime}\right)$ induces a vector for all $\left(\varphi_{n}: n \in+\right) \in L(\mathbb{Z}(+))$ inducing $\varphi$.

TheOREM 2.7. With the notation above, $\hat{f}=\operatorname{ind}_{n \in,}\left(\hat{f}_{n}\right)$.

The essence of Theorem 2.7 has been known since the 1930's. We have been unable to derive it from any results we have found in the literature, but we suspect that it would yield to general principles of a Hilbert space approach to spectral methods as in, for instance, Picard [17, Section 2.2].

THEOREM 2.8. For all $t \in \mathbb{R}, \hat{f}^{[t]}=\operatorname{ind}_{n \in,},\left(\hat{f}_{n}^{[l]}\right)$.

§3. Proofs. For each $j \in \mathbb{N}$ and $n \in A$, let $h_{n, j}:=\operatorname{res}_{n}\left(h_{j}\right)$ and $\lambda_{n, j}:=$ $4-2 \pi(2 j+1) / n$.

Lemma 3.1. Given $j \in \mathbb{N}$, and $\xi \in \mathbb{R}(+)$ and $n \in t$ with $n \geqslant \rho(\xi)$, then

$$
\left|\left(\left(\hat{s}_{n}-\lambda_{n, j}\right) h_{n, j}\right)(\xi(n))\right|=O\left(n^{-9 / 4}\right) .
$$

Proof. Let $x:=\xi(n)$. Taylor's Theorem gives

$$
h_{j}(\xi+\delta)=\sum_{\alpha=0}^{\infty} \delta^{\alpha} h_{j}^{(\alpha)}(\xi) / \alpha !
$$

Putting $\delta= \pm v(n)^{-2}$, and using the fact that each function $r \mapsto\left|h_{j}^{(\alpha)}(r)\right|$ is bounded above, we have

$$
\begin{aligned}
& h_{n, j}(x-1)-2 h_{n, j}(x)+h_{n, j}(x+1) \\
&= v(n)^{-5} h_{j}^{\prime \prime}(\xi)+\sum_{\alpha=4}^{\alpha}\left(1+(-1)^{\alpha}\right) v(n)^{-2 \alpha-1} h_{j}^{(\alpha)}(\xi) / \alpha ! \\
&= v(n)^{-5} h_{j}^{\prime \prime}(\xi)+O\left(v(n)^{-9}\right) .
\end{aligned}
$$

Similar manipulations give

$$
(-2+2 \cos 2 \pi x / n) h_{n, j}(x)=-\xi^{2} v(n)^{-5} h_{j}(\xi)+O\left(v(n)^{-9}\right),
$$

whence

$$
\left(\left(\hat{s}_{n}-\lambda_{n, j}\right) h_{n, j}\right)(x)+O\left(n^{-9 / 4}\right)=v(n)^{-5}\left(h_{j}^{\prime \prime}(\xi)+\left(-\xi^{2}+2 j+1\right) h_{j}(\xi)\right)=0 .
$$

Given any vector $v$ in an inner product space, we write the modulus of $v$ as $\|v\|$. In particular, for $\psi \in L^{2}(\mathbb{R})$, we write $\|\psi\|:=\|\psi\|_{2}$.

Lemma 3.2. Given $j \in \mathbb{N}$ and $n \in t$, then $\left\|\left(\hat{s}_{n}-\lambda_{n, j}\right) h_{n, j}\right\|=o\left(n^{-3 / 2}\right)$.

Proof. Let $k_{n}:=\left(\hat{s}_{n} \lambda_{n, j}\right) h_{n, j}$. By Lemma 3.1,

$$
\sum_{x \in \mathbb{Z}(n):|x| \leq v(n)^{2} \log n}\left|k_{n}(x)\right|^{2}=O\left(n^{-4} \log n\right) .
$$


Choose $B \in \mathbb{R}$ such that $\left|h_{j}(r)\right|<B r^{j} e^{-r^{2} / 2}$ for all $r \in \mathbb{R}$. For $x \in \mathbb{Z}(n)$ and $\xi=$ $x v(n)^{-2}$, we have $v(n)\left|k_{n}(x)\right| \leqslant 8 B \xi^{j} e^{-\xi^{2} / 2}$ because $\left|\lambda_{n, j}\right| \leqslant 4$ and $\hat{s}_{n}$ is a sum of four unitary operators. So

$$
\sum_{x \in \mathbb{Z}(n) ;|x|>v(n)^{2} \log n}\left|k_{n}(x)\right|^{2} \leqslant 64 B^{2} \int_{r:|r|+1 \geqslant \log n} r^{2 j} e^{-r^{2}} d r .
$$

The right-hand expression diminishes faster than $n^{-4} \log n$, and so $\left\|k_{n}\right\|=$ $o\left(n^{-2} \log n\right)$.

LEMMA 3.3. Given $j \in \mathbb{N}$, and sufficiently large $n \in 1$, then $\hat{s}_{n}$ has an eigenvalue in the open interval $(4-4 \pi j / n-3 \pi / n, 4-4 \pi j / n-\pi / n)$.

Proof. Suppose, for a contradiction, that there exists an infinite subset $A^{\prime \prime}$ of . ' such that, for each $n \in f^{\prime}$, there is no eigenvalue of $\hat{s}_{n}$ in the specified interval. Since the function $h_{j}$ has precisely $j$ zeros, we cannot have $\left\|h_{n, j}\right\|=0$ unless $n \leqslant j$. So we may define $g_{n, j}:=h_{n, j} /\left\|h_{n, j}\right\|$ for all $n \in$, ' with $n>j$. Lemma 2.3 gives $\lim _{n \in,},\left\|h_{h, j}\right\|=\left\|h_{j}\right\|=1$, and then Lemma 3.2 tells us that

$$
\left\|\left(\hat{s}_{n}-(4-4 \pi j / n-2 \pi / n)\right) g_{n, j}\right\|=o\left(n^{-3 / 2}\right) .
$$

For each $n \in f$ ' with $n>j$, the hypothesis on . ' ' allows us to write $g_{n, j}=$ $a_{n}+b_{n}$, where $a_{n}$ is a linear combination of eigenvectors of $\hat{s}_{n}$ with associated eigenvalues at least $4-4 \pi j / n-\pi / n$, and $b_{n}$ is a linear combination of eigenvectors of $\hat{s}_{n}$ with associated eigenvalues at most $4-4 \pi j / n-3 \pi / n$. Using the fact that $a_{n}$ and $b_{n}$ are orthogonal, we obtain the contradiction

$$
\left\|\left(\hat{s}_{n}-(4-4 \pi j / n-2 \pi / n)\right)\left(a_{n}+b_{n}\right)\right\| \geqslant \pi / n\left(\left\|a_{n}\right\|^{2}+\left\|b_{n}\right\|^{2}\right)=\pi / n .
$$

The next five results concern the following scenario: let $F \in \mathbb{R}$, and for each $n \in .$, , let $\omega_{n}$ be a real unit eigenvector of both $\hat{s}_{n}$ and $\hat{f}_{n}$ such that the eigenvalue of $\hat{s}_{n}$ associated with $\omega_{n}$ has the form $4-2 \pi E_{n} / n$ where $E_{n} \leqslant F$. Thus $\omega_{n}$ satisfies Harper's Equation

$$
\omega_{n}(x-1)+\omega_{n}(x+1)=2\left(1-\pi E_{n} / n-\cos 2 \pi x / n\right) \omega_{n}(x)
$$

for $x \in \mathbb{Z}$. Since $\hat{s}_{n}=\hat{u}_{n}+\hat{u}_{n}^{\dagger}+\hat{v}_{n}+\hat{v}_{n}^{\dagger}$ as a sum of four unitary operators which do not mutually commute, we have $E_{n}>0$, and, in particular, $F>0$.

LEMMA 3.4. Let $n \in 1$, and let $x \in \mathbb{Z}(n)$ such that $\cos 2 \pi x / n \leqslant 1-\pi E_{n} / n$. If $x>0$, then $\left|\omega_{n}(x)\right| \leqslant\left|\omega_{n}(x-1)\right|$. If $x<0$, then $\left|\omega_{n}(x)\right| \leqslant\left|\omega_{n}(x+1)\right|$.

Proof. Since $\omega_{n}$ is an eigenvector of $\hat{f}_{n}^{2}$, we have $\left|\omega_{n}(z)\right|=\left|\omega_{n}(-z)\right|$ for all $z \in \mathbb{Z}$. So, if the assertion fails, then there must exist some $x \in \mathbb{Z}$ such that $\cos 2 \pi x / n \leqslant 1-\pi E_{n} / n$ and $\left|\omega_{n}(x-1)\right|<\left|\omega_{n}(x)\right|>\left|\omega_{n}(x+1)\right|$. But Harper's Equation forces $\left|\omega_{n}(x-1)+\omega_{n}(x+1)\right| \geqslant 2\left|\omega_{n}(x)\right|$.

LEMMA 3.5. Given sufficiently large $n \in$. , , and $x \in \mathbb{Z}(n)$, then $v(n)\left|\omega_{n}(x)\right| \leqslant(2 F)^{1 / 4}$. 
Proof. For each $n \in, 1$, let $M_{n}$ be the maximum value of $\left|\omega_{n}(x)\right|$ with $x \in$ $\mathbb{Z}$. Let us fix an element $n \in 1$, and assume that $n$ is sufficiently large for our purposes. Write $M:=M_{n}$, and choose $y \in \mathbb{Z}(n)$ such that $\omega_{n}(y)=M$. Since $\left|\omega_{n}(y)\right|=\left|\omega_{n}(-y)\right|$, we may insist that $y<0$. We assume that $\omega_{n}(y)>0$; the argument when $\omega_{n}(y)<0$ is similar. Since $n$ is sufficiently large, Lemma 3.4 and the condition $y<0$ ensure that

$$
1 \geqslant \cos 2 \pi(y+x) / n>1-\pi E_{n} / n \geqslant 1-\pi F / n
$$

for each integer $z$ with $0 \leqslant z \leqslant \sqrt{n / \pi F}$. For each such $z$, let $\Delta(z):=$ $\omega_{n}(y+z)-\omega_{n}(y+z-1)$. We have $|\Delta(z+1)-\Delta(z)| \leqslant 2 \pi F M / n$, hence $|\Delta(z)| \leqslant$ $2 \pi F M z / n$ and

$$
\omega_{n}(y+z) \geqslant M-\sum_{x=0}^{z} 2 \pi F M x / n=M(1-\pi F z(z+1) / n)>M\left(1-\pi F(z+1)^{2} / n\right) .
$$

Letting $m$ be the largest integer such that $m \leqslant \sqrt{n / \pi F}$, then

$$
\begin{aligned}
1 & =\left\|\omega_{n}\right\|^{2} \geqslant \sum_{z=0}^{m-1} \omega_{n}(y+z)^{2}>M^{2} \sum_{z=1}^{m}\left(1-\pi F z^{2} / n\right)^{2} \\
& =M^{2}\left(M-\frac{2 \pi F}{n}\left(\frac{m^{3}}{3}+\frac{m^{2}}{2}+\frac{m}{6}\right)+(\pi F / n)^{2}\left(\frac{m^{5}}{5}+\frac{m^{4}}{2}+\frac{m^{3}}{3}-\frac{m}{30}\right)\right) .
\end{aligned}
$$

Now letting the element $n \in .1$ vary, we have

$$
1>M_{n}^{2}(8 \sqrt{n / \pi F} / 15+o(\sqrt{n}))>M_{n}^{2}\left(v(n)^{2} / \sqrt{2 F}+o\left(v(n)^{2}\right)\right) .
$$

We now construct a function $\omega,: \mathbb{R}(f) \rightarrow \mathbb{R}$. Let $n_{0}$ be the smallest element of 1 . Given an element $\zeta \in \mathbb{R}(.+)$ with $\rho(\zeta) \leqslant n_{0}$, then Lemma 3.5

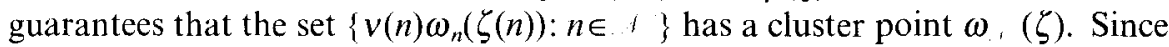
there are only finitely many $\zeta \in \mathbb{R}(1)$ with $\rho(\zeta) \leqslant n_{0}$, there exists an infinite

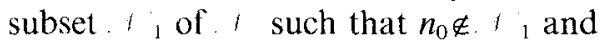

$$
\omega,(\zeta)=\lim _{n \in, 1}\left(v(n) \omega_{n}(\zeta(n))\right)
$$

for all $\zeta \in \mathbb{R}($,$) with \rho(\zeta) \leqslant n_{0}$.

Now let $t \geqslant 1$, and assume, inductively, that we have defined $A_{s}$ for all integers $s$ with $1 \leqslant s \leqslant t$. For each $s$, let $n_{s}$ be the smallest element of $t s$. We

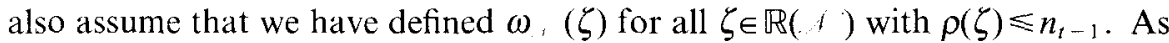
before, there exists an infinite subset. $t_{i+1}$ of $t_{t}$ such that $n_{t} \notin \lambda_{t+1}$, and the real number

$$
\omega,(\zeta)=\lim _{n \in i_{1+1}}\left(v(n) \omega_{n}(\zeta(n))\right)
$$

is a cluster point of $\left\{v(n) \omega_{n}(\zeta(n)): n \in t,\right\}$ for all $\zeta \in \mathbb{R}(t)$ with $\rho(\zeta) \leqslant n_{t}$. Letting. $1_{*}:=\left\{n_{t}: t \in,\right\}$, then

$$
\omega,(\zeta)=\lim _{n \in .,}\left(v(n) \omega_{n}(\zeta(n))\right)
$$

for all $\zeta \in \mathbb{R}(+)$. 
LEMMA 3.6. Given $\eta \in \mathbb{R}(1)$ with $\eta^{2} \geqslant 4 F$, then $\omega,(\eta)^{2} \leqslant 4 F /|\eta|^{3}$.

Proof. Given real $r>0$, and $n \in t$, let $\Omega_{n}(r):=\sum_{x \in Z} \omega_{n}(x)^{2}$ and $\Omega_{n}^{\prime}(r):=\sum_{x \in Z^{\prime}} \omega_{n}(x)^{2}$, where $Z:=\left\{x \in \mathbb{Z}(n):|x| \leqslant r v(n)^{2}\right\}$ and $Z^{\prime}:=\{x \in \mathbb{Z}(n)$ : $\left.|x|>r v(n)^{2}\right\}$. Then

$$
\Omega_{n}(r)+\Omega_{n}^{\prime}(r)=\left\|\omega_{n}\right\|^{2}=1 .
$$

Now $\hat{s}_{n}=\hat{u}_{n}+\hat{u}_{n}^{\dagger}+\hat{v}_{n}+\hat{v}_{n}^{\dagger}$ as a sum of four unitary operators, so

$$
4-2 \pi F / n \leqslant\left\langle\omega_{n}\left|\hat{s}_{n}\right| \omega_{n}\right\rangle=\left\langle\omega_{n}\left|\hat{u}_{n}+\hat{u}_{n}^{\dagger}\right| \omega_{n}\right\rangle+\left\langle\omega_{n}\left|\hat{v}_{n}+\hat{v}_{n}^{\dagger}\right| \omega_{n}\right\rangle
$$

and both of the terms of the sum have absolute value at most 2 . Therefore

$$
\begin{aligned}
1-\pi F / n & \leqslant\left\langle\omega_{n}\left|\hat{u}_{n}+\hat{u}_{n}^{\dagger}\right| \omega_{n}\right\rangle / 2=\sum_{x \in \mathbb{Z}(n)} \omega_{n}(x)^{2} \cos 2 \pi x / n \\
& \leqslant \Omega_{n}(r)+\Omega_{n}^{\prime}(r) \cos 2 \pi r v(n)^{2} / n=1-\Omega_{n}^{\prime}(r) \pi r^{2} / n+O\left(n^{-2}\right) .
\end{aligned}
$$

We deduce that $\Omega_{n}^{\prime}(r)<F / r^{2}+O(1 / n)$. We may assume that $\eta>0$. Let $n \in$. be such that $n \geqslant \rho(\eta)$. Writing $y:=\eta(n)$, then

$$
\cos \pi y / n+O\left(n^{-2}\right)<1-\pi \eta^{2} / 4 n \leqslant 1-\pi E_{n} / n .
$$

By Lemma 3.4, $\left|\omega_{n}(x)\right|$ is monotonically decreasing in the range $\chi \in \mathbb{Z} \cap[y /$ 2,y]. Recalling that $\omega_{n}(x)^{2}=\omega_{n}(-x)^{2}$, and summing $\omega_{n}(x)^{2}$ over the integers $x \in[-y,-y / 2] \cup[y / 2, y]$, we obtain $\omega_{n}(y)^{2}(y+O(1)) \leqslant \Omega_{n}^{\prime}(\eta / 2)$. Lemma 3.5 implies that $\omega_{n}(y)^{2}=O\left(n^{-1 / 2}\right)$, so that

$$
\omega_{n}(y)^{2} \eta v(n)^{2}+O\left(n^{-1 / 2}\right)<4 F / \eta^{2} .
$$

The assertion now follows from the construction of $\omega$, .

We allow $\mathbb{R}(\uparrow)$ to inherit the subspace topology from $\mathbb{R}$.

LEMmA 3.7. The function $\omega,: \mathbb{R}(1) \rightarrow \mathbb{R}$ is continuous.

Proof. Let $\xi, \eta \in \mathbb{R}(\mathcal{A})$ with $\xi \geqslant \eta$. We shall calculate an upper bound for $\omega,(\xi)-\omega,(\eta)$. Let $n \in \mathcal{A}$, and assume that $n$ is large; in particular, $\rho(\xi) \leqslant n \geqslant \rho(\eta)$. Let $x:=\xi(n)$ and $y:=\eta(n)$. The eigenvalue $i_{n}$ of $\hat{f}_{n}$ associated with $\omega_{n}$ is a 4 th root of unity, and

$$
\begin{aligned}
i_{n}\left(\omega_{n}(x)-\omega_{n}(y)\right) & =\left(\hat{f}_{n} \omega_{n}\right)(x)-\left(\hat{f}_{n} \omega_{n}\right)(y) \\
& =\frac{1}{\sqrt{n}} \sum_{z \in \mathbb{Z}(n)} \omega_{n}(z)\left(e^{2 \pi i x z / n}-e^{2 \pi i y z / n}\right) \\
& =\frac{-2 i}{\sqrt{n}} \sum_{z \in \mathbb{Z}(n)} \omega_{n}(z) e^{\pi i(x+y) z / n} \sin \pi(x-y) z / n
\end{aligned}
$$

Therefore

$$
v(n)^{2}\left|\omega_{n}(x)-\omega_{n}(y)\right| \leqslant \sqrt{\frac{2}{\pi}} \sum_{z \in \mathbb{Z}(n)}\left|\omega_{n}(z) \sin \pi(x-y) z / n\right| .
$$


Let $\tau \in \mathbb{R}($ ( ) ) be such that $\tau \geqslant 2 \sqrt{F}$. Insisting that $n \geqslant \rho(\tau)$, let $t:=\tau(n)$. Let $Z:=\{x \in \mathbb{Z}:|x| \leqslant t\}$, and $Z^{\prime}:=\mathbb{Z}(n)-Z$. Bearing in mind that $n$ is large, the proof of Lemma 3.6 shows that

$$
\left|\omega_{n}(z)\right|<\sqrt{5 F / \zeta^{3}} / v(n)=v(n)^{2} \sqrt{5 F} z^{-3 / 2},
$$

where $z \in Z^{\prime}$ and $\zeta:=z v(n)^{-2}$. Therefore

$$
\begin{aligned}
\sum_{z \in Z^{\prime}}\left|\omega_{n}(z) \sin \pi(x-y) z / n\right| & <2 v(n)^{2} \sqrt{5 F} \sum_{z=t+1}^{\infty} z^{-3 / 2} \\
& \leqslant 6 v(n)^{2} \sqrt{F} \int_{t}^{\infty} r^{-3 / 2} d r \\
& =12 v(n)^{2} \sqrt{F / t} \\
& =12 v(n) \sqrt{F / \tau} .
\end{aligned}
$$

On the other hand, via Lemma 3.5,

$$
\begin{aligned}
\sum_{z \in Z}\left|\omega_{n}(z) \sin \pi(x-y) z / n\right| \\
\quad \leqslant \frac{(2 F)^{1 / 4}}{v(n)} \sum_{z=-1}^{t}\left|\pi(x-y) z / n+O\left(n^{-3 / 2}\right)\right| \\
\leqslant(2 F)^{1 / 4}\left(\pi(x-y) t(t+1) / n+O\left(n^{-1 / 2}\right)\right) / v(n) \\
<F^{1 / 4}\left((x-y) t^{2} / v(n)+O\left(n^{-3 / 2}\right)\right) \\
=F^{-1 / 4} v(n) \tau^{2}(\zeta-\eta)+O\left(n^{-3 / 2}\right) .
\end{aligned}
$$

Therefore,

hence

$$
v(n)\left|\omega_{n}(x)-\omega_{n}(y)\right| \leqslant \sqrt{2 / \pi}\left(F^{1 / 4} \tau^{2}(\zeta-\eta)+12 \sqrt{F / \tau}\right)+O(1 / n),
$$

$$
\left|\omega,(\zeta)-\omega_{n}(\eta)\right|<F^{1 / 4} \tau^{2}(\zeta-\eta)+12 \sqrt{F / \tau}
$$

Lemma 3.8. The function $\omega, \mathbb{R}(+) \rightarrow \mathbb{R}$ extends uniquely to a realvalued continuous function $\omega: \mathbb{R} \rightarrow \mathbb{C}$. Furthermore, $\omega$ is a uniformly continuous function in $L^{2}(\mathbb{R})$, and $\|\omega\|=1$.

Proof. The first part is immediate from Lemma 3.7. By Lemmas 3.5 and 3.6, the function $r \mapsto|\omega(r)|$ is bounded by both $(2 F)^{1 / 4}$ and $O\left(|r|^{-3 / 2}\right)$. So $\omega$ is uniformly continuous.

To prove the last part, we may assume that $\mathscr{F}=A^{*}$. Let $\tau \in \mathbb{R}(\mathcal{H})$ with $\tau \geqslant \sqrt{4 F}$. Lemma 3.6 gives $\omega(r)^{2} \leqslant 4 F /|r|^{3}$ whenever $|r| \geqslant \tau$. In particular,

$$
\lim _{\tau \rightarrow \infty} \int_{|r|<\tau} \omega(r)^{2} d r=0 .
$$


The proof of Lemma 3.6 shows that

$$
\lim _{\tau \rightarrow \infty} \lim _{n \in .} \sum_{x \in \mathbb{Z}(n):|x|>\tau v(n)^{2}} \omega_{n}(x)^{2}=0 .
$$

Let us now fix $\tau$. Since each $\left\|\omega_{n}\right\|=1$, it suffices to show that

$$
\int_{-\tau}^{\tau} \omega(r)^{2} d r=\lim _{n \in .} \sum_{x \in Z} \omega_{n}(x)^{2},
$$

where $Z:=\left\{x \in \mathbb{Z}(n):|x| \leqslant \tau v(n)^{2}\right\}$. Choose any real $\varepsilon>0$. The proof of Lemma 3.7 shows that, given sufficiently large $n \in, 1$, then for all $\xi, \xi^{\prime} \in \mathbb{R}(+1)$ with $\left|\xi-\xi^{\prime}\right|<\delta$ we have

$$
\left|v(n)^{2}\left(\omega_{n}(\xi(n))^{2}-\omega\left(\xi^{\prime}(n)\right)^{2}\right)\right|<\varepsilon / 2 .
$$

For each $\zeta \in \mathbb{R}(1) \cap[-\tau, \tau]$, we have

$$
\left|v(n)^{2} \omega_{n}(\zeta(n))^{2}-\omega(\zeta)^{2}\right|<\varepsilon / 2
$$

for sufficiently large $n$. So, for each such $\zeta$, there exists some neighbourhood $U(\zeta)$ of $\zeta$ in $[-\tau, \tau]$ such that, for all $r \in U(\zeta)$, we have

$$
\left|\omega(r)^{2}-\omega(\zeta)^{2}\right|<\varepsilon
$$

and, furthermore, given sufficiently large $n$, then for all $\xi \in \mathbb{R}(. /) \cap U(\zeta)$ with $\rho(\xi) \leqslant n$, we have

$$
\left|v(n)^{2} \omega_{n}(\xi(n))^{2}-\omega(\zeta)^{2}\right|<\varepsilon .
$$

Let $\left\{U\left(\zeta_{\alpha}\right): \alpha \in A(\varepsilon)\right\}$ be a finite subcover of $[-\tau, \tau]$, and write $[-\tau, \tau]=$ $\bigcup_{\alpha \in A(\varepsilon)} V(\varepsilon, \alpha)$, as a disjoint union, where each $V(\varepsilon, \alpha)$ is a connected subset of $U\left(\zeta_{\alpha}\right)$. We have a Lebesgue integral

$$
\int_{-\tau}^{\tau} \omega(r)^{2} d r=\lim _{\varepsilon \rightarrow 0} \sum_{\alpha \in A(\varepsilon)} \mu(V(\varepsilon, \alpha)) \omega\left(\zeta_{\alpha}\right)^{2} .
$$

where $\mu$ denotes the Borel measure. Since there are $O(\sqrt{n})$ elements $x$ of $\mathbb{Z}(n)$ satisfying $|x| \leqslant \tau v(n)^{2}$, we have

$$
\begin{aligned}
\sum_{\alpha \in A(\varepsilon)} \mu(V(\varepsilon, \alpha)) \omega\left(\zeta_{\alpha}\right)^{2}+\varepsilon O(1) & =\sum_{\alpha \in A(\varepsilon)}\left(\sum_{x \in Z(n): x V(n)^{2} \in V(\varepsilon, \alpha)} \omega_{n}\left(x^{2}\right)\right) \\
& =\sum_{x \in Z} \omega_{n}(x)^{2}
\end{aligned}
$$

for sufficiently large $n$.

LEMmA 3.9. Given $j \in \mathbb{N}$, and sufficiently large $n \in .$, , then $\hat{s}_{n}$, has a unique eigenvalue in the closed interval $[4-4 \pi(j+1) / n, 4-4 \pi j / n]$.

Proof. Thanks to Lemma 3.3, it suffices to derive a contradiction from the assumption that, for infinitely many $n \in$, , there exist two orthogonal real 
unit eigenvectors $\omega_{n}^{\prime}$ and $\omega_{n}^{\prime \prime}$ of $\hat{s}_{n}$ whose associated eigenvalues lie in the specified interval. We replace,, with the set of such $n$. For each $n \in$, ${ }^{\prime}$, we put either $\omega_{n}=\omega_{n}^{\prime}$ or else $\omega_{n}=\omega_{n}^{\prime \prime}$, insisting that $\left\langle\omega_{n} \mid h_{n, j}\right\rangle \leqslant 3 / 4$ for sufficiently large $n$. By Lemma 3.2, $\lim _{n \in,}\left\langle\omega_{n} \mid h_{n, j^{\prime}}\right\rangle^{2}=0$ for all $j^{\prime} \in \mathbb{N}$ with $j^{\prime} \neq j$. Since $\left\{h_{j}: j \in \mathbb{N}\right\}$ is an orthonormal basis for $L^{2}(\mathbb{R})$, we have $\sum_{k \in \mathbb{N}}\left\langle\omega \mid h_{k}\right\rangle^{2}=1$. The desired contradiction will be achieved when we have shown that

$$
\left\langle\omega \mid h_{k}\right\rangle=\lim _{n \in,}\left\langle\omega_{n} \mid h_{n, k}\right\rangle .
$$

The argument is similar to the proof of Lemma 3.8; we shall outline the steps.

Using Lemma 3.6, and the fact that $h_{j}$ is rapidly decreasing, it is easy to reduce to the task of proving that

$$
\int_{-\tau}^{\tau} \omega(r) h_{k}(r) d r=\lim _{n \in,} \sum_{x \in,} \omega_{n}(x) h_{n, j}(x),
$$

where $\tau$ and $Z$ are as in the proof of Lemma 3.8. For each $\zeta \in \mathbb{R}(.1) \cap[-\tau, \tau]$, we choose a neighbourhood $U^{\prime}(\zeta)$ of $\zeta$ such that, for all $r \in U^{\prime}(\zeta)$, we have

$$
\left|\omega(r) h_{k}(r)-\omega(\zeta) h_{k}(\zeta)\right|<\varepsilon,
$$

and furthermore, given sufficiently large $n \in t$, then, for all $\xi \in \mathbb{R}(A) \cap U(\zeta)$ with $\rho(\xi) \leqslant n$, we have

$$
\left|v(n)^{2} \omega_{n}(\xi(n)) h_{n, k}(\xi(n))-\omega(\zeta) h_{k}(\zeta)\right|<\varepsilon .
$$

Choosing a finite subcover $\left\{U^{\prime}\left(\zeta_{\alpha}^{\prime}\right): \alpha \in A^{\prime}(\varepsilon)\right\}$, and writing $[-\tau, \tau]=$ $\bigcup_{\alpha \in A^{\prime}(\varepsilon)} V^{\prime}(\varepsilon, \alpha)$ just as before, we have

$$
\sum_{\alpha \in A^{\prime}(\varepsilon)} \mu\left(V^{\prime}(\varepsilon, \alpha)\right) \omega\left(\zeta_{\alpha}^{\prime}\right) h_{k}\left(\zeta_{\alpha}^{\prime}\right)+\varepsilon O(1)=\sum_{x \in Z} \omega_{n}(x) h_{n, k}(x)
$$

Again, the assertion follows by letting $n \rightarrow \infty$ and then letting $\varepsilon \rightarrow 0$.

Proof of Theorem 2.5. For each $n \in .1$, let us enumerate the elements $\sigma_{n, 0}^{\prime}, \sigma_{n, 1}^{\prime}, \ldots, \sigma_{n, n-1}^{\prime}$ of the basis $S_{n}$ such that the associated eigenvalues $\lambda_{n, 0}^{\prime}, \lambda_{n, 1}^{\prime}, \ldots, \lambda_{n, n-1}^{\prime}$ are in decreasing order (perhaps not strictly). Lemmas 3.3 and 3.9 imply that, given any $j \in \mathbb{N}$, then for sufficiently large $n \in t$, we have

$$
4-4 \pi j / n-3 \pi / n<\lambda_{n, j}^{\prime}<4-4 \pi j / n-\pi / n \text {. }
$$

Lemma 3.9 also implies that, for such given $j$ and chosen $n$, we may write $h_{n, j}=\alpha_{n, j}+c_{n, j} \sigma_{n, j}^{\prime}+\beta_{n, j}$, where $c_{n, j} \in \mathbb{C}$, and $\alpha_{n, j}$ is a linear combination of eigenvectors of $\hat{s}_{n}$ with associated eigenvalues at least $4-4 \pi j / n$, and $\beta_{n, j}$ is a linear combination of eigenvectors of $\hat{s}_{n}$ with associated eigenvalues at most $4-4 \pi(j+1) / n$. We saw in the proof of Lemma 3.3 that $\left\|h_{n, j}\right\| \rightarrow 1$ as $n$ increases, so that Lemma 3.2 gives $\lim _{n \in,}\left\|\alpha_{n, j}\right\|=\lim _{n \in, .}\left\|\beta_{n, j}\right\|=0$, and $\lim _{n \in,},\left|c_{n, j}\right|=1$. But $h_{n, j}$ and $\sigma_{n, j}^{\prime}$ both have real coordinates with respect to the bases $\left\{x^{*}: x \in \mathbb{Z}(n)\right\}$ of $L(\mathbb{Z}(n))$; furthermore, if $j$ is even, then $h_{n, j}(0)>0$, while if $j$ is odd, then $h_{n, j}(0)=0<h_{n, j}(1)$. Therefore $\lim _{n \in}, c_{n, j}=1$. For fixed $j \in \mathbb{N}$ and sufficiently large $n \in .1$, we have shown that $\sigma_{n, j}^{\prime}=\sigma_{n, j}$. It follows 
that, when $n$ is sufficiently large, $\sigma_{n, j}=\left(h_{n, j}-\alpha_{n, j}-\beta_{n, j}\right) / c_{n, j}$. Given any uniformly continuous $\varphi \in L^{2}(\mathbb{R})$, Lemma 2.3 now gives

$$
\lim _{n \in .}\left\langle\operatorname{res}_{n}(\varphi) \mid \sigma_{n, j}\right\rangle=\lim _{n \in}\left\langle\operatorname{res}_{n}(\varphi) \mid h_{n, j}\right\rangle=\left\langle\varphi \mid h_{j}\right\rangle .
$$

Proof of Theorem 2.6. The proof of Theorem 2.5 shows that

$$
\lim _{n \in,}\left\langle h_{n, j} \sigma_{n, k}\right\rangle=\delta_{j, k}
$$

for all $j, k \in \mathbb{N}$. Write $\psi=\sum_{j \in \mathbb{N}} c_{j} h_{j}$ with each $c_{j} \in \mathbb{C}$. For each $n \in$., , let $\psi_{n}:=\sum_{j=0}^{n-2} c_{j} \sigma_{n, j}$. Let $\mathscr{f}(\mathbb{R})$ denote the subspace of $\gamma(\mathbb{R})$ consisting of the linear combinations $\theta=\sum_{j \in \mathbb{N}} d_{j} h_{j}$ with only finitely many of the $d_{j}$ non-zero. For each such $\theta$, we have

$$
\langle\theta \mid \psi\rangle=\sum_{j \in \mathbb{N}} d_{j} c_{j}=\lim _{n \in .}\left\langle\operatorname{res}_{n}(\theta) \mid \psi_{n}\right\rangle .
$$

The assertion follows because $\bar{T}(\mathbb{R})$ is dense in the space of uniformly continuous functions in $L^{2}(\mathbb{R})$.

Proof of Theorem 2.7. This is similar to the proof of Theorem 2.8 below.

Proof of Theorem 2.8. Let $\varphi, \psi \in L^{2}(\mathbb{R})$ be such that $\varphi$ is uniformly continuous. Let $\left(\psi_{n}: n \in t\right) \in L(\mathbb{Z}(t))$ be such that $\psi=\operatorname{ind}_{n \in},\left(\psi_{n}\right)$. Let $t \in \mathbb{R}$, and write $\hat{g}:=\hat{f}^{[t]}$. For each $n \in, t$, write $\hat{g}_{n}:=\hat{f}_{n}^{[t]}$. We are to prove that

$$
\langle\varphi \mid \hat{g} \varphi\rangle=\lim _{n \in .}\left\langle\operatorname{res}_{n}(\varphi) \mid \hat{g}_{n} \psi_{n}\right\rangle .
$$

Since $\bar{f}(\mathbb{R})$ is dense in $L^{2}(\mathbb{R})$, we may assume that $\psi \in, \quad r(\mathbb{R})$. In fact, we may assume that $\varphi=h_{j}$ for some $j \in \mathbb{N}$. Write $\psi=\sum_{k \in \mathbb{N}} c_{k} h_{k}$. For each $n \in 1$, write $\psi_{n}=\sum_{k} c_{n, k} \sigma_{n, k}$, where the index $k$ runs over the range specified in Section 2. Now

$$
c_{j}=\left\langle h_{j} \mid \psi\right\rangle=\lim _{n \in,}\left\langle h_{n, j} \mid \psi_{n}\right\rangle=\lim _{n \in,} c_{n, j} .
$$

Putting $\varepsilon:=e^{2 \pi i j t}$, then $\left\langle h_{j} \mid \hat{g} \psi\right\rangle=\varepsilon c_{j}=\lim _{n \in}, \varepsilon c_{n, j}=\lim _{n \in},\left\langle h_{i, j} \mid \hat{g}_{n} \psi_{n}\right\rangle$.

\section{References}

1. G. G. Athanasiu and E. G. Floratos. Coherent states in infinite quantum mechanics. Nuclear Phys. B, 425 (1994), 343-364.

2. G. G. Athanasiu, E. G. Floratos and S. Nicolis. Holomorphic quantization on the torus and finite quantum mechanics. J. Phys. A, 29 (1996), 6737-6745.

3. N. M. Atakishiev and S. K. Suslov. Difference analogues of the harmonic oscillator. (English translation.) Teoreticheskaya i Mat. Fiz., 85 (1990), 64-73.

4. R. Balian and C. Itzykson. Observations sur la mécanique quantique finie. C. R. Acad. Sc. Paris, Ser. I, 303 (1986), 773-778.

5. L. Barker. Phase space over an abelian group of odd order (preprint).

6. L. Barker. Continuum quantum systems as limits of discrete quantum systems, l: state vectors. J. Functional Analysis 186 (2001), 153-166.

7. L. Barker, C. Candan, T. Hakioğlu, A. Kutay and H. M. Özaktaş. The discrete harmonic oscillator, Harper's equation, and the discrete fractional Fourier transform. J. Phys. A: Math. Gen., 33 (2000), 2209-2222. 
8. C. Candan. The Discrete Fractional Fourier Transform (Bilkent University M.S. thesis, July 1998, unpublished).

9. C. Candan, M. A. Kutay and H. M. Özaktaş. The discrete fractional Fourier transform. Proceedings of the 1999 IEEE International Conference on Acoustics, Speech, and Signal Processing, Vol IIl (IEEE, Pistcataway, New Jersey 1999), 1713-1716.

10. T. Hakioğlu. Linear canonical transformations and quantum phase: a unified canonical and algebraic approach. J. Phys. A: Math. Gen., 32 (1999), 4111-4130.

11. A. C. McBride and F. H. Kerr. On Namias' fractional Fourier transform. IMA J. of Applied Math., 39 (1987), 159 - 175.

12. J. H. McClellen and T. W. Parks. Eigenvalue and eigenvector decomposition of the discrete Fourier transform. IEEE Trans. Audio Electroacoust., AU-20 (1972), 66-74.

13. V. Namias. The fractional order Fourier transform and its application to quantum mechanics. J. Inst. Math. Applics., 25 (1980), 241-265.

14. H. M. Özaktaş. M. A. Kutay and D. Mendlovic. Introduction to the fractional Fourier transform and its applications. In ed. P. W. Hawkes, Advances in Imaging and Electron Physics, Vol 106 (Academic Press, San Diego, California, 1998), 239-291.

15. H. M. Özaktaş, D. Mendlovic, M. A. Kutay and Z. Zalevsky, The Fractional Fourier Transform: with Applications to Optics and Signal Processing (Wiley, New York, to appear).

16. S.-C. Pei and M.-H. Yeh. Improved discrete fractional Fourier transform. Optics Lett., 22 (1997), $1047-1049$.

17. R. Picard. Hilbert Space Approach to Some Classical Transforms, Pitman Research Notes in Math., 196 (Longman, Harlow, U.K., 1989).

18. R. Rammal and J. Bellisard. An algebraic semi-classical approach to Bloch electrons in a magnetic field. J. Phys. France, 51 (1990), 1803-1830.

19. M. S. Richman, T. W. Parks and R. G. Shenoy. Discrete-time, discrete-frequency, time frequency analysis. IEEE Trans. Signal Processing, 46 (1998), 1517-1527.

20. J. H. Wilkinson. The Algebraic Eigenvalue Problem, Oxford Univ. Press (1965).

Dr. L. Barker,

Department of Mathematics,

Bilkent University,

06533 Bilkent,

Ankara,

Turkey.

E-mail: barker $(a$ fen.bilkent.edu.tr
42C15: FOURIER ANALYSIS; Nontrigonometric Fourier analysis; Series of general orthogonal functions, generalized Fourier expansions, nonorthogonal expansions.

Received on the 29 th of April, 1999. 